Pacific Journal of Mathematic

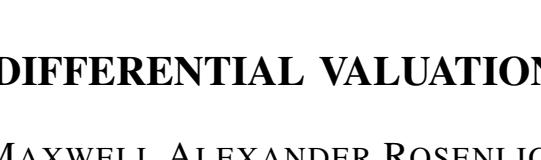




\title{
DIFFERENTIAL VALUATIONS
}

\section{MAXWELL ROSENLICHT}

Dedicated to Gerhard Hochschild on the occasion of his 65th birthday

\begin{abstract}
A notion of "differential valuation" is defined for ordinary differential fields of characteristic zero by postulating for a given valuation of the field a natural analogue of the elementary L'Hospital's rule. Such valuations occur implicitly in classical analysis, for example in Hardy's orders of infinity and in the study of singular points of systems of ordinary differential equations. The fundamental properties of differential valuations are worked out in this paper, numerous examples are discussed, and it is shown that a differential valuation can always be extended to an algebraic extension field. Applications are anticipated to the study of singularities of algebraic differential equations.
\end{abstract}

In the following, $k$ will denote an ordinary differential field of characteristic zero, ' its derivation, and $C$ its subfield of constants.

THEOREM 1. Let $k$ be a differential field of characteristic zero with subfield of constants $C, v$ a valuation of $k$ that induces the trivial valuation on $C$, and let $\mathfrak{b}$ and $\mathfrak{m}$ be respectively the valuation ring of $v$ and its maximal ideal. Then the following statements are equivalent:

(1) If $a \in \mathfrak{p}, b \in \mathfrak{m}, b \neq 0$, then $a^{\prime} b / b^{\prime} \in \mathfrak{m}$.

(2) If $a, b \in k^{*}$ and $0<v(a) \leqq v(b)$, then $\left(b / a-b^{\prime} / a^{\prime}\right) \in \mathfrak{m}$.

(3) If $a, b \in k^{*}$ and $v(a) \leqq v(b)<0$, then $\left(b / a-b^{\prime} / a^{\prime}\right) \in \mathfrak{m}$.

(4) If $a \in \mathfrak{0}, b \in k^{*}, 1 / b \in \mathfrak{m}$, then $a^{\prime} b / b^{\prime} \in \mathfrak{m}$.

First note that $b \in \mathfrak{m}$ if and only if $v(b)>0$. Also, $C \cap \mathfrak{m}=\{0\}$, so that if $b \in \mathfrak{m}$ and $b \neq 0$, then $b^{\prime} \neq 0$.

Proof that $(1) \Rightarrow(2)$. Under the assumptions of (2) we can write $b=a c$, with $c \in \mathfrak{D}$. Since $a \in \mathfrak{m}$ we get

$$
\frac{b}{a}-\frac{b^{\prime}}{a^{\prime}}=c-\frac{a^{\prime} c+a c^{\prime}}{a^{\prime}}=-\frac{c^{\prime} a}{a^{\prime}},
$$

and by (1) this last quantity is is $\mathrm{m}$.

Proof that $(2) \Rightarrow(3)$. Under the assumptions of (3), if we write $\alpha=1 / a, \beta=1 / b$, we get $0<v(\beta) \leqq v(\alpha)$, so that $0<v(\alpha) \leqq v\left(\alpha^{2} / \beta\right)$. Hence

$$
\frac{b}{a}-\frac{b^{\prime}}{a^{\prime}}=\frac{(1 / \beta)}{(1 / \alpha)}-\frac{(1 / \beta)^{\prime}}{(1 / \alpha)^{\prime}}=\frac{\alpha}{\beta}-\frac{\alpha^{2} \beta^{\prime}}{\beta^{2} \alpha^{\prime}}=\frac{\left(\alpha^{2} / \beta\right)^{\prime}}{\alpha^{\prime}}-\frac{\alpha^{2} / \beta}{\alpha},
$$


which is in $\mathfrak{m}$, by (2).

Proof that $(3) \Rightarrow(4)$. Making the assumptions of (4) and also replacing $a$ by $a+1$ if necessary to reduce to the special case in which $a$ is a unit in $\mathfrak{D}$, we have $v(b)=v(a b)<0$, so that $(a b) / b-$ $(a b)^{\prime} / b^{\prime} \in \mathfrak{m}$, giving $a^{\prime} b / b^{\prime} \in \mathfrak{m}$.

Proof that $(4) \Rightarrow(1)$. This follows immediately from the identity $b / b^{\prime}=-(1 / b) /(1 / b)^{\prime}$.

The picture to bear in mind, to be more fully explained below in Examples 1 and 2 , is that in which $k$ consists of differentiable real or complex valued functions on some domain in $C$, which is $\boldsymbol{R}$ or $C$, as the case may be, differentiation meaning differentiation with respect to the given variable of $C$, with the condition that as the variable approaches a certain fixed limit, finite or infinite, each function in $k$ approaches a limit, which is either in $C$ or the symbol $\infty$, and $\mathfrak{D}$ is the set of all functions in $k$ whose limits are in $C$. Then (2) and (3) are simply the usual $0 / 0$ and $\infty / \infty$ versions of L'Hospital's rule. In a slight variant of this rule, if $a, b \in k^{*}$ both have limit 0 or limit $\infty$ (i.e., $v(a), v(b)$ are either both positive or both negative) and if one of the two quantities $b / a, b^{\prime} / a^{\prime}$ has limit $\infty$, then so does the other; this extra bit of L'Hospital's rule comes from (2) and (3) with $a$ and $b$ interchanged.

COROLLARY 1. Under the same assumptions as in the theorem, each of the statements (1), (2), (3), (4) implies

(5) If $a, b \in k^{*}$ and $v(a), v(b) \neq 0$, then $v(a) \leqq v(b)$ if and only if $v\left(a^{\prime}\right) \leqq v\left(b^{\prime}\right)$.

Conversely, under the same assumptions, if $\mathfrak{v}=C+\mathfrak{m}$, then (5) implies (1), (2), (3), (4).

For the proof, instead of (5) consider the equivalent statement

$\left(5^{\prime}\right)$ If $a, b \in k^{*}$ and $v(a), v(b) \neq 0$, then $(v(a)=v(b)) \Rightarrow\left(v\left(a^{\prime}\right)=\right.$ $\left.v\left(b^{\prime}\right)\right)$ and $(v(a)<v(b)) \Rightarrow\left(v\left(a^{\prime}\right)<v\left(b^{\prime}\right)\right)$.

We first show that any of the equivalent statements (1), (2), (3), (4) implies $\left(5^{\prime}\right)$. Statement $\left(5^{\prime}\right)$ is implied by $(2)$ if $0<v(a) \leqq v(b)$ and it is implied by (3) if $v(a) \leqq v(b)<0$. In the remaining case $v(a)<$ $0<v(b)$, use $v\left((1 / a)^{\prime}\right)=v\left(-a^{\prime} / a^{2}\right)=v\left(a^{\prime}\right)-2 v(a)>v\left(a^{\prime}\right)$ and $v\left((1 / b)^{\prime}\right)=$ $v\left(b^{\prime}\right)-2 v(b)<v\left(b^{\prime}\right)$. If $v(1 / a) \leqq v(b)$, then (2) implies that $v\left(b^{\prime}\right) \geqq$ $v\left((1 / a)^{\prime}\right)>v\left(a^{\prime}\right)$. If $v(1 / a)>v(b)$, we have $v(a)<v(1 / b)<0$, so that (3) implies $v\left(a^{\prime}\right)<v\left((1 / b)^{\prime}\right)<v\left(b^{\prime}\right)$. Thus (1), (2), (3), (4) each imply $\left(5^{\prime}\right)$. Conversely, assume $\left(5^{\prime}\right)$ and suppose first that $a, b \in \mathfrak{m}, a, b \neq 0$. Then $0<v(b)<v(a b)$, whence $v\left(b^{\prime}\right)<v\left((a b)^{\prime}\right)$. But also $v\left(b^{\prime}\right)<v\left(b^{\prime} a\right)$, 
so that $v\left(b^{\prime}\right)<v\left((a b)^{\prime}-b^{\prime} a\right)=v\left(a^{\prime} b\right)$. Hence $v\left(a^{\prime} b / b^{\prime}\right)>0$, or $a^{\prime} b / b^{\prime} \in \mathfrak{m}$. This last conclusion also holds if instead of assuming $a \in \mathfrak{m}$ we just suppose $a \in \mathfrak{D}$, provided $\mathfrak{o}=C+\mathfrak{m}$, and hence statement (1) holds.

In the context of Theorem 1, statements (1) and (4) say that $v\left(a^{\prime} b / b^{\prime}\right)>0$, or $v\left(a^{\prime}\right)>v\left(b^{\prime} / b\right)$. This implies that the subset $\left\{v\left(a^{\prime}\right)\right.$ : $a \in \mathfrak{D}, a \notin C\}$ of the value group $v\left(k^{*}\right)$ is bounded from below and the subset $\left\{v\left(b^{\prime} / b\right): b \in k^{*}, v(b) \neq 0\right\}$ is bounded from above. The next result says that the set $\left\{v\left(a^{\prime}\right): a \in k, a \notin C\right\}$ is bounded neither from above nor from below.

COROLLARY 2. In the context of the theorem and its various equivalent conditions, if $v$ is nontrivial then for any $a \in k^{*}$ there exist $x, y \in k^{*}$ such that $v\left(x^{\prime}\right)>v(a)>v\left(y^{\prime}\right)$.

Fix some $b \in \mathfrak{0}, b \notin C$. Then for any $y \in k^{*}$ such that $v(y) \neq 0$ we have $b^{\prime} y / y^{\prime} \in \mathfrak{m}$, or $v\left(b^{\prime} y / y^{\prime}\right)>0$, or $v\left(y^{\prime}\right)<v\left(b^{\prime} y\right)$. If we choose $y \in k$ such that $v(y)<v\left(a / b^{\prime}\right), v(y) \neq 0$, then $v\left(y^{\prime}\right)<v(a)$, as desired. To get our desired $x$, assume, as we may, that $v(a) \neq 0$, and note that if $u \in k^{*}, v(u)>0$, then $v\left(u^{\prime} a / a^{\prime}\right)>0$, or $v\left(u^{\prime}\right)>v\left(a^{\prime} / a\right)$. Therefore $v\left(\left(u^{2}\right)^{\prime}\right)=v\left(2 u u^{\prime}\right)=v(u)+v\left(u^{\prime}\right)>v(u)+v\left(a^{\prime} / a\right)$. If we take $x=u^{2}$, with $v(u)>\max \left\{0, v\left(a^{2} / a^{\prime}\right)\right\}$, then we get $v\left(x^{\prime}\right)>v(a)$.

Definition. Let $k$ be a differential field of characteristic zero, $C$ its subfield of constants. A differential valuation of $k$ is a valuation $v$ of $k$ that is trivial on $C$ and such that if $\mathfrak{o}$ and $\mathfrak{m}$ are respectively its valuation ring and maximal ideal, then $\mathfrak{D}=C+\mathfrak{m}$ and $(a \in \mathfrak{p}, b \in$ $\mathfrak{m}, b \neq 0) \Rightarrow\left(a^{\prime} b / b^{\prime} \in \mathfrak{m}\right)$.

Thus a valuation $v$ on the differential field $k$ with constant subfield $C$ is a differential valuation of $k$ if the equivalent conditions of Theorem 1 hold and the natural embedding of $C$ into the residue class field $\mathrm{o} / \mathrm{m}$ is surjective.

EXAMPLE 1. Here $k$ is an arbitrary Hardy field (cf. [1, p. 107]) containing the real numbers $\boldsymbol{R}$. We recall that a Hardy field is a set of germs of differentiable real-valued functions on neighborhoods of $+\infty$ in $\boldsymbol{R}$ that is closed under differentiation and form a field with respect to the usual germ addition and multiplication. Any nonzero element of $k$ has a reciprocal, hence only a finite number of zeros, therefore is ultimately of constant sign. The same being true of the derivative of any nonconstant element of $k$, each element of $k$ is ultimately monotonic. Thus for each $f \in k, \lim _{x \rightarrow+\infty} f(x)$ exists as an element of $\boldsymbol{R}$ or as one of the symbols $+\infty$ or $-\infty$. The differential field structure of $k$ is given and its valuation is that 
associated with the place $f \mapsto \lim _{x \rightarrow+\infty} f(x)$ (identifying $+\infty$ and $-\infty$ ), whose valuation ring consists of all $f \in k$ having finite limit at $+\infty$. That this valuation is a differential valuation of $k$ follows from L'Hospital's rule.

Essentially the same theory holds for germs of real-valued differentiable functions on deleted right (or left) sided neighborhoods in $\boldsymbol{R}$ of any given point of $\boldsymbol{R}$. For example, we can consider differential fields of functions on neighborhoods of $0+$ in $R$; in this case by a switch in variable from $x$ to $1 / x$ we get the previous theory, but with another differentiation operation, the two differentiations differing only by the nonvanishing factor $-1 / x^{2}$.

We remark in passing that many specific examples of Hardy fields may be obtained by starting with the field of rational functions $\boldsymbol{R}(x)$ and constructing a chain of extension fields, each extension field being obtained from its predecessor by the adjunction of the exponential, or of the logarithm of the absolute value, of an element of the predecessor field.

EXAMPLE 2. Let $k$ be a field of germs of differentiable complexvalued functions on neighborhoods of $+\infty$ in $\boldsymbol{R}$. Suppose that $k$ contains $C$, is closed under differentiation, and that the following condition is satisfied:

(A) For each $f \in k, \lim _{t \rightarrow+\infty} f(t)$ exists, as an element of $C \cup\{\infty\}$.

As in Example 1, we have a valuation of $k$ whose valuation ring is the set of all elements $f$ of $k$ such that $\lim _{t \rightarrow+\infty} f(t)$ is finite. Suppose that the following condition also holds:

(B) For each $f \in k$ such that $\lim _{t \rightarrow+\infty} f(t)=0$, there exists $M \in \boldsymbol{R}$ such that for any sufficiently large $t_{1}, t_{2} \in \boldsymbol{R}$ with $t_{1}<t_{2}$, we have

$$
\int_{t_{1}}^{t_{2}}\left|f^{\prime}(t)\right| d t \leqq M\left|f\left(t_{1}\right)-f\left(t_{2}\right)\right|
$$

Then our valuation of $k$ is a differential valuation. To verify this, we must show that if $f, g \in k$ are such that $f(t)$ and $g(t)$ approach 0 as $t \rightarrow+\infty$ and $g^{\prime} \neq 0$, then $f(t) / g(t)$ and $f^{\prime}(t) / g^{\prime}(t)$ have the same limits as $t \rightarrow+\infty$. It suffices to prove this statement when $f^{\prime}(t) / g^{\prime}(t)$ has a finite limit $c$, and indeed, upon replacing $f$ by $f-c g$, it suffices to prove that

$$
\begin{aligned}
& \left(f, g \in k, g^{\prime} \neq 0, \text { and } f(t), g(t), f^{\prime}(t) / g^{\prime}(t) \text { all approach } 0\right. \text { as } \\
& t \longrightarrow+\infty) \Longrightarrow(f(t) / g(t) \text { approaches } 0 \text { as } t \longrightarrow+\infty) .
\end{aligned}
$$

To prove this last statement, suppose the contrary, so that there exists $a>0$ such that $|f(t) / g(t)|>a$ for $t$ sufficiently large. Let $M>0$ be associated with $g$ by the property of (B). Let $T_{1}>0$ be 
such that $f(t), g(t), f^{\prime}(t), g^{\prime}(t)$ are all defined, with $g(t), g^{\prime}(t) \neq 0$ and $\left|f^{\prime}(t) / g^{\prime}(t)\right|<a / M$ for all $t \geqq T_{1}$, such that $\left|f\left(T_{1}\right) / g\left(T_{1}\right)\right|>a$, and such that for any $T_{2}>T_{1}$ we have $\int_{T_{1}}^{T_{2}}\left|g^{\prime}(t)\right| d t \leqq M\left|g\left(T_{1}\right)-g\left(T_{2}\right)\right|$. Since $\left|f\left(T_{1}\right) / g\left(T_{1}\right)\right|>a$ and $f(t), g(t)$ approach 0 as $t \rightarrow+\infty$, we can choose $T_{2}>T_{1}$ such that $\left|\left(f\left(T_{1}\right)-f\left(T_{2}\right)\right) /\left(g\left(T_{1}\right)-g\left(T_{2}\right)\right)\right|>a$. For each $\tau \epsilon$ $\left[T_{1}, T_{2}\right]$ we have $\lim _{t \rightarrow \tau}(f(t)-f(\tau)) /(g(t)-g(\tau))=f^{\prime}(\tau) / g^{\prime}(\tau)$, so that for $|t-\tau|$ sufficiently small we have $|f(t)-f(\tau)| \leqq(a / M)|g(t)-g(\tau)|$. Since $\left[T_{1}, T_{2}\right]$ is compact, we can find $t_{0}, t_{1}, \cdots, t_{n}$ such that $T_{1}=$ $t_{0}<t_{1}<\cdots<t_{n}=T_{2}$ and such that for each $i=1, \cdots, n$ we have $\left|f\left(t_{i-1}\right)-f\left(t_{i}\right)\right| \leqq(a / M)\left|g\left(t_{i-1}\right)-g\left(t_{i}\right)\right|$. Then

$$
\begin{aligned}
a\left|g\left(T_{1}\right)-g\left(T_{2}\right)\right| & <\left|f\left(T_{1}\right)-f\left(T_{2}\right)\right|=\left|\sum_{i=1}^{n}\left(f\left(t_{i-1}\right)-f\left(t_{i}\right)\right)\right| \\
& \leqq \sum_{i=1}^{n}\left|f\left(t_{i-1}\right)-f\left(t_{i}\right)\right| \leqq \sum_{i=1}^{n}(a / M)\left|g\left(t_{i-1}\right)-g\left(t_{i}\right)\right| \\
& =(a / M) \sum_{i=1}^{n}\left|\int_{t_{i-1}}^{t_{i}} g^{\prime}(t) d t\right| \\
& \leqq(a / M) \int_{T_{1}}^{T_{2}}\left|g^{\prime}(t)\right| d t \leqq a\left|g\left(T_{1}\right)-g\left(T_{2}\right)\right|
\end{aligned}
$$

a contradiction. This completes the proof that under conditions (A) and (B) our valuation of $k$ is a differential valuation.

Some remarks on the practical utility of $(\mathrm{A})$ and $(\mathrm{B})$ are in order. First, condition (A) is easily shown to be satisfied when there exist $f_{1}, f_{2}, \cdots$ in $k^{*}$ such that $k=C\left(f_{1}, f_{2}, \cdots\right)$ and for any nonconstant power product $f$ of $f_{1}, f_{2}, \cdots$ we have $\lim _{t-+\infty} f(t)$ either 0 or $\infty$. Second, condition (B) may be difficult to verify in a practical example, but there is a useful alternate condition, going back to (1) of Theorem 1 , which is necessary and sufficient for our valuation of $k$ to be a differential valuation: if $f_{1}, f_{2}, \cdots$ are as in the above test for (A), then for any nonconstant power products $f$ and $g$ of $f_{1}, f_{2}, \cdots$ that approach 0 as $t \rightarrow+\infty$, also $f^{\prime} g / g^{\prime}$ approaches 0 as $t \rightarrow+\infty$. (This last condition is clearly necessary, and its sufficiency is easily checked by a direct computation that also occurs in the proof of Theorem 2 below. Indeed the sufficiency of this last condition is the special case of Theorem 2 in which the items $K, k, \mathscr{C}, C, T$ occurring there are the present $k, C, C, C$ and multiplicative group generated by $\boldsymbol{C}^{*}$ and $f_{1}, f_{2}, \cdots$.) Specific cases where this procedure is applicable are provided below at the end of Example 10 and in Example 13.

Example 2 is applicable to the circumstance in which we have a field $k$ of meromorphic functions at some point $z_{0} \in C$, with $k$ containing $C$ and closed under differentiation, and there is a continuously differentiable path $\gamma:[0,1] \rightarrow C$ from $z_{0}=\gamma(0)$ to $z_{1}=\gamma(1)$ such that each element of $k$ can be analytically continued along $\gamma$ from $z_{0}$ up 
to any point $\gamma(t), 0<t<1$, and approaches a limit, either in $C$ or the symbol $\infty$, as $z \rightarrow z_{1}$ along $\gamma$, and an appropriate analogue of (B) holds for the restriction to the path $\gamma$ of the continuations of the elements of $k$. As a matter of fact, the differential valuation of $k$ that is thus obtained does not depend on $\gamma$, any path with the same endpoints and sufficiently close to $\gamma$ in a suitable sense giving the same differential valuation, at least in the case where $k$ is finitely generated over $\boldsymbol{C}$. This example and numerous special cases lead one to believe that the proper way of dealing with the never-preciselydefined classical notion of "singularity of a system of ordinary differential equations" is through differential valuations.

EXAMPLE 3. If $C$ is a field of characteristic zero and $z$ an indeterminate, the formal power series field $C((z))$ is a differential field under the usual derivation (which is trivial on $C$, continuous in the topology of $C((z))$, and has $\left.z^{\prime}=1\right)$, and the usual valuation of $C((z))$ (trivial on $C$, continuous, and discrete) is a differential valuation of $C((z))$.

In the special case of the preceding Example 2 in which $\gamma$ is the constant path $\gamma(t)=z_{0}=0$, power series expansion in powers of $z$ gives an embedding of differential fields $k \subset C((z))$. If $f_{1}, f_{2} \in k$ and $v\left(f_{1}\right)=v\left(f_{2}\right) \neq 0$, then $\lim _{z \rightarrow 0} f_{1}(z) / f_{2}(z)=\lim _{z \rightarrow 0} f_{1}^{\prime}(z) / f_{2}^{\prime}(z)$ is simply the ratio of leading coefficients.

EXAMPLE 4. Let us see how certain classical computations go through in a perfectly general differential field $k$ with differential valuation $v$. Suppose that $x, y \in k^{*}$ and that $y$ is an exponential of $x$, so that $y^{\prime} / y=x^{\prime}$. Suppose that $v(x) \geqq 0$. Then since $v\left(x^{\prime} y / y^{\prime}\right)=0$ we must have $v(y)=0$. Choosing $x_{0}, y_{0} \in C$ so that $v\left(x-x_{0}\right)>0$, $v\left(y-y_{0}\right)>0$, we have $y_{0} \neq 0$. Induction on $n$ using L'Hospital's rule shows that for each $n \geqq 0$

$$
\frac{y-y_{0}\left(1+\frac{x-x_{0}}{1 !}+\cdots+\frac{\left(x-x_{0}\right)^{n}}{n !}\right)}{\left(x-x_{0}\right)^{n+1}}
$$

is in the valuation ring of $v$ and has residue class field image $y_{0} /(n+1)$ !. Thus the partial sums of the series $y_{0} \exp \left(x-x_{0}\right)$ give finer and finer approximations to $y$, in the usual sense of asymptotic developments. If $k=C(x, y)$ we can even embed $k$ in the formal power series field $C\left(\left(x-x_{0}\right)\right)$ so that we have actual equality $y=$ $y_{0} \exp \left(x-x_{0}\right)$.

EXAMPLE 5 . Let $k$ be a differential field, $v$ a differential valuation 
of $k$, and $a \in k^{*}$. Then $k$ has another differential field structure, with the same subfield of constants, in which the new derivative of any $u \in k$ is $a u^{\prime}$. The old $v$ is a differential valuation of the new differential field.

ExAMPLE 6 . Let $C$ be any field of characteristic zero, $k$ a finite extension field of $C$ of transcendence degree one such that $C$ is algebraically closed in $k$. Fix a nonzero $C$-derivation' of $k$. Then $k$ is a differential field and $C$ its subfield of constants. Let $v$ be the valuation of $k$ associated with any place of $k$ over $C$ that is rational over $C$. Then $v$ is a differential valuation of $k$. To see this, note that the $C$-derivations of $k$ form a vector space over $k$ of dimension one, so by Example 5 we may replace' by $d / d t$, where $t$ is a uniformizing variable for the place in question, i.e., $t \in k$ and $v(t)=1$ (if $v\left(k^{*}\right)$ is normalized to be $\boldsymbol{Z}$ ). Then we have a natural embedding of differential fields $k \subset C((t))$ preserving the valuation and the derivation, that is we have a differential subfield of the field of Example 3.

EXAMPLE 7. Consider the nonconvergent formal power series $y=\sum_{n=0}^{\infty} n ! x^{n} \in \boldsymbol{R}((x))$. This satisfies the differential equation

$$
x^{2} y^{\prime}+(x-1) y=-1,
$$

so that the subfield $\boldsymbol{R}(x, y)$ of $\boldsymbol{R}((x))$ is a differential subfield, and we have a differential valuation on this subfield induced by the differential valuation of $\boldsymbol{R}((x))$ of Example 3 . To interpret this, note that the differential equation has the real-variable solutions

$$
-\frac{1}{x e^{1 / x}} \int \frac{e^{1 / x} d x}{x}
$$

taking the arbitrary constant of integration into the integral sign. We have the formal identity

$$
\begin{aligned}
-\frac{1}{x e^{1 / x}} \int \frac{e^{1 / x} d x}{x} & =1+1 ! x+2 ! x^{2}+\cdots+n ! x^{n} \\
& -\frac{1}{x e^{1 / x}} \int(n+1) ! x^{n} e^{1 / x} d x .
\end{aligned}
$$

As $x \rightarrow 0+$ we have $x^{N} e^{1 / x} \rightarrow+\infty$ for all $N \in Z$. Interpreting $\int$ above to mean $\int_{a}^{x}$, for some fixed $a>0$, we get a solution of the differential equation on the real interval $(0,+\infty)$ which has the given series $\sum_{n=0}^{\infty} n ! x^{n}$ as an asymptotic expansion at $0+$. Any other solution of the differential equation on $(0,+\infty)$ differs from the present solution by a constant multiple of $1 /\left(x e^{1 / x}\right)$ and has the same asymptotic 
expansion. On the other hand, as $x \rightarrow 0$ - we have $e^{1 / x} / x^{N} \rightarrow 0$ for all $N \in Z$. It turns out that the differential equation has precisely one solution on the interval $(-\infty, 0)$ which has a finite limit at $0-$ and this solution is obtained by interpreting $\int$ to mean $\int_{0-}^{x}$. This solution has the given asymptotic expansion $\sum_{n=0}^{\infty} n ! x^{n}$ at $0-$.

EXAMPLE 8. In [2], Kolchin defines a valued differential field to be a differential field $k$ with a valuation $v$ such that the set $\left\{v\left(a^{\prime} / a\right): a \in k^{*}, v(a) \neq 0\right\}$ is bounded from above and from below, and he uses such fields to prove an important diophantine result. If $k$ is a differential field with a differential valuation $v$, then the set $\left\{v\left(a^{\prime} / a\right): a \in k^{*}, v(a) \neq 0\right\}$ is bounded from above, by (1) and (4). It will be seen as a consequence of Theorem 4 that this set is in fact finite, therefore a fortiori bounded, if $\Gamma \boldsymbol{\otimes}_{z} \boldsymbol{Q}$ is a finite dimensional vector space over $\boldsymbol{Q}, \Gamma$ being the value group $v\left(k^{*}\right)$, a case that includes all the most useful examples. On the other hand, for a general differential field $k$ with differential valuation $v$ the set $\left\{v\left(a^{\prime} / a\right): a \in k^{*}, v(a) \neq 0\right\}$ need not be bounded from below: a counterexample is the Hardy field $k=\boldsymbol{R}(x, \exp x, \exp (\exp x), \cdots)$, as $x \rightarrow$ $+\infty$. There are also valued differential fields for which the valuation is not a differential valuation in the present sense: an example is the differential field of meromorphic functions on $C$ given by $k=$ $C\left(z, \mu(z), f^{\prime}(z)\right)$, where $z$ is the complex variable, $z^{\prime}=1, \mu(z)$ is the Weierstrass $\mu$-function corresponding to some given period lattice in $C$, with valuation ring the set of all elements of $k$ of the form

$$
\frac{a_{0}+a_{1} z^{-1}+a_{2} z^{-2}+\cdots}{1+b_{1} z^{-1}+b_{2} z^{-2}+\cdots}
$$

where $a_{0}, a_{1}, a_{2}, \cdots, b_{1}, b_{2}, \cdots$ are in the differential subfield $\boldsymbol{C}(\mu(z)$, $\left.f^{\prime}(z)\right)$ of $k$ and $a_{0}(0) \neq \infty$.

EXAMPLE 9. There is no differential valuation $v$ of the differential field $\boldsymbol{C}\left(\boldsymbol{z}, \mu^{\prime}(\boldsymbol{z}), \boldsymbol{z}^{\prime}(\boldsymbol{z})\right.$ ) (where $\mu(\boldsymbol{z})$ is a Weierstrass $\mu$-function of the complex variable $z$, as in Example 8) such that $v(z)<0$. For suppose that $v$ is a valuation of $C\left(z, f(z), \mu^{\prime}(z)\right)$ with residue field $C$ such that $v(z)<0$. Then $v$ induces a valuation on the elliptic function field $C\left(f^{\prime}(z), \mu^{\prime}(z)\right)$ that corresponds to some point $z_{0} \in C$, so that $v\left(\mu\left(z-z_{0}\right)\right)<0$. We know that $C\left(\mu(z), \mu^{\prime}(z)\right)=C\left(\mu\left(z-z_{0}\right), \mu^{\prime}\left(z-z_{0}\right)\right)$. If $/(z)$ satisfies the equation

$$
\left(\kappa^{\prime}(z)\right)^{2}=4(k(z))^{3}-g_{2} /(z)-g_{3},
$$

with $g_{2}, g_{3} \in C$, and we set $t(z)=\mu(z) / \mu^{\prime}(z)$, we compute 


$$
t^{\prime}(z)=1-\frac{12(/ /(z))^{2}-g_{2}}{8(/ /(z))^{3}-2 g_{2 / 4}(z)-2 g_{3}} .
$$

Since $\mu\left(z-z_{0}\right)$ satisfies the same differential equation as $\mu(z)$, we get $v\left(t\left(z-z_{0}\right)\right)>0, v\left(t^{\prime}\left(z-z_{0}\right)\right)=0$. Hence $v\left(t^{\prime}\left(z-z_{0}\right) \dot{z} / z^{\prime}\right)=v(z)<0$, so that $v$ is not a differential valuation.

The following result will have many applications.

THEOREM 2. Let $K$ be a differential field of characteristic zero, $k$ a differential subfield of $K, \mathscr{C}$ a subfield of the field of constants of $K$, and $v$ a valuation of $K$ that is trivial on $\mathscr{C}$. Suppose that $C=$ $\mathscr{C} \cap k$ maps surjectively to the image of $k$ in the residue class field of $v$. Let $T$ be a subgroup of the multiplicative group $K^{*}$ such that $k^{*} \subset T$, such that $K=\mathscr{C}(T)$, such that any constant in $T$ is in $C$, such that $(a \in T, v(a)=0) \Rightarrow\left(a \in k^{*}\right)$, and such that if $a, b \in T$ and $v(a), v(b)>0$ then $v\left(a^{\prime} b / b^{\prime}\right)>0$. Then $v$ is a differential valuation of $K$, and $\mathscr{C}$ is the field of constants of $K$.

It will be convenient to first prove the following preliminary result.

LeMma. Under the conditions of Theorem 2, each nonzero element $x \in \mathscr{C}[T]$ can be written as a finite sum $x=\sum_{i=1}^{n} \xi_{i} t_{i}$, with each $\xi_{i} \in$ $\mathscr{C}^{*}$, each $t_{i} \in T$, and $v\left(t_{1}\right)<\left(t_{2}\right)<\cdots<v\left(t_{n}\right)$.

It is trivial that $x$ can be written in the form $x=\sum_{i=1}^{n} \xi_{i} t_{\imath}$, with each $\xi_{i} \in \mathscr{C}^{*}$ and each $t_{\imath} \in T$. Write $x$ in this way, and suppose that $n$ is minimal for such a representation of $x$. We may suppose that $v\left(t_{1}\right)=v\left(t_{2}\right)=\cdots=v\left(t_{r}\right)<v\left(t_{r+1}\right), \cdots, v\left(t_{n}\right)$. We have, by our assumptions, $t_{i} / t_{1} \in k$ for $i=1, \cdots, r$. Clearly $v(x) \geqq v\left(t_{1}\right)$. If $v(x)>v\left(t_{1}\right)$, using the equation $x / t_{1}=\sum_{i=1}^{n} \xi_{2}\left(t_{i} / t_{1}\right)$ and mapping into the residue class field of $v$ gives a relation of linear dependence over $C$ among $\xi_{1}, \cdots, \xi_{r}$. Say that $\xi_{r}=c_{1} \xi_{1}+\cdots+c_{r-1} \xi_{r-1}$, with $c_{1}, \cdots, c_{r-1} \in C$. Then $x=\xi_{1}\left(t_{1}+c_{1} t_{r}\right)+\cdots+\xi_{r-1}\left(t_{r-1}+c_{r-1} t_{r}\right)+\xi_{r+1} t_{r+1}+\cdots+\xi_{n} t_{n}$. Since $t_{i} / t_{1} \in k$ for $i=1, \cdots, r$, each of the coefficients $t_{1}+c_{1} t_{r}, \cdots, t_{r-1}+$ $c_{r-1} t_{r}$ is in $T \cup\{0\}$, and we get an expression for $x$ of the type $\sum \xi_{2} t_{i}$ of length less than $n$, which is impossible. Thus $v(x)=v\left(t_{1}\right)$. For $i=1, \cdots, r$, let $d_{\imath} \in C$ have the same residue class field image as $t_{i} / t_{1}$. Then $x=\left(\sum_{i=1}^{r} \xi_{2} d_{i}\right) t_{1}+\sum_{i=2}^{r} \xi_{i}\left(t_{\imath}-d_{i} t_{1}\right)+\xi_{r+1} t_{r+1}+\cdots+\xi_{n} t_{n}$. Now $\sum_{i=1}^{r} \xi_{i} d_{i} \in \mathscr{C}$ and for $i=2, \cdots, r$ we have $t_{i}-d_{i} t_{1} \in k$ and $v\left(t_{i}-d_{i} t_{1}\right)>v(x)$. Hence we could have assumed that in the representation of minimal length $x=\sum_{i=1}^{n} \xi_{i} t_{i}$, with each $\xi_{i} \in \mathscr{C}$ and each $t_{i} \in T$, we had $v(x)=v\left(t_{1}\right)<v\left(t_{2}\right), \cdots, v\left(t_{n}\right)$. Applying this result to the representation of minimal length of $x-\xi_{1} t_{1}$ as $x-\xi_{1} t_{1}=\sum_{i=2}^{n} \xi_{2} t_{i}$, we can 
assume that $v\left(x-\xi_{1} t_{1}\right)=v\left(t_{2}\right)<v\left(t_{3}\right), \cdots, v\left(t_{n}\right)$. Continuing thus, we get the lemma.

We now prove the theorem. Since $K$ is the field of quotients of $\mathscr{C}[T]$, the lemma implies that any element $u \in K^{*}$ can be written as

$$
u=u_{0}\left(\sum_{i=1}^{n} \xi_{i} a_{i}\right) /\left(\sum_{j=1}^{m} \eta_{j} b_{j}\right),
$$

with $u_{0} \in T$, each $\xi_{i}, \eta_{j} \in \mathscr{C}^{*}$, each $a_{i}, b_{j} \in T, a_{1}=b_{1}=\eta_{1}=1$ and $v\left(a_{i}\right)$, $v\left(b_{j}\right)>0$ for $i, j>1$. In this case $v(u)=v\left(u_{0}\right)$, and if $v(u)=0$ then $u_{0} \in k$ and $u$ has the same residue class field image as does $u_{0} \xi_{1}$. Thus the residue class field of $v$ is $\mathscr{C}$. If $v(u) \neq 0$, then $v\left(u_{0}\right) \neq 0$, so $u_{0} \notin$ $C$ and $u_{0}^{\prime} \neq 0$. Thus if $v(u) \neq 0$, we can write

$$
\frac{u^{\prime}}{u}=\frac{u_{0}^{\prime}}{u_{0}}\left(1+\frac{\sum_{i=2}^{n} \xi_{i} a_{i}^{\prime} u_{0} / u_{0}^{\prime}}{\sum_{i=1}^{n} \xi_{i} a_{i}}-\frac{\sum_{j=2}^{m} \eta_{j} b_{j}^{\prime} u_{0} / u_{0}^{\prime}}{\sum_{j=1}^{m} \eta_{j} b_{j}}\right) .
$$

If $v\left(u_{0}\right)>0$, we get $v\left(a_{i}^{\prime} u_{0} / u_{0}^{\prime}\right), v\left(b_{j}^{\prime} u_{0} / u_{0}^{\prime}\right)>0$ for each $i, j>1$ by our assumptions, and we get the same conclusions if $v\left(u_{0}\right)<0$ by using the identity $u_{0} / u_{0}^{\prime}=-\left(1 / u_{0}\right) /\left(1 / u_{0}\right)^{\prime}$. Thus if $v(u) \neq 0$, we have $v\left(u^{\prime} / u\right)=v\left(u_{0}^{\prime} / u_{0}\right)$. Hence if $v(u) \neq 0$, we have $v(u)=v\left(u_{0}\right), v\left(u^{\prime}\right)=$ $v\left(u_{0}^{\prime}\right)$. We now show that if $u \in K$ is constant, then $u \in \mathscr{C}$. If $u \neq$ 0 is constant, so is $1 / u$, so we may suppose $v(u) \geqq 0$. Subtracting from $u$ a suitable element of $\mathscr{C}$, we may suppose $v(u)>0$, in which case we already know that $u^{\prime} \neq 0$. Thus the constants of $K$ are precisely $\mathscr{C}$. Since for each $u \in K^{*}$ such that $v(u) \neq 0$ there is a $u_{0} \in T$ such that $v(u)=v\left(u_{0}\right), v\left(u^{\prime}\right)=v\left(u_{0}^{\prime}\right)$, the condition (1) of Theorem 1 is true for elements of $K$ since the corresponding facts hold for elements of $T$, and this completes the proof.

EXAMPLE 10. Let $C$ be a field of characteristic zero, $c_{1}, \cdots, c_{r}$ elements of $C$ that are linearly independent over $\boldsymbol{Q}$, let $y_{1}, \cdots, y_{r}$ be indeterminates over $C$, and let $K$ be the differential field $C\left(y_{1}, \cdots, y_{r}\right)$, with differentiation defined as trivial on $C$ and such that $y_{i}^{\prime} / y_{i}=c_{i}$ for $i=1, \cdots, r$. For any $n_{1}, \cdots, n_{r} \in Z$, we have

$$
\left(y_{1}^{n_{1}} \cdots y_{r}^{n}\right)^{\prime} /\left(y_{1}^{n_{1}} \cdots y_{r}^{n_{r}}\right)=n_{1} c_{1}+\cdots+n_{r} c_{r},
$$

which is nonzero unless $n_{1}=\cdots=n_{r}=0$. Let $\tau$ be any isomorphic embedding of the subgroup $\boldsymbol{Z} c_{1}+\cdots+\boldsymbol{Z} c_{r}$ of $C$ into an ordered abelian group. Then there is a unique valuation $v$ of $K$ that is trivial on $C$ and such that $v\left(y_{i}\right)=\tau\left(c_{i}\right)$ for each $i=1, \cdots, r$. Apply the last theorem to the present field $K$ and valuation $v$, with $k=$ $\mathscr{C}=C$ and $T$ the multiplicative subgroup of $K$ generated by $C^{*}$ 
and $y_{1}, \cdots, y_{r}$. We conclude that $C$ is the subfield of constants of $K$ and $v$ a differential valuation. Note that any differential valuation of the differential field $K$ such that $v\left(y_{1}\right) \neq 0$ can be obtained in this way, since for $i=2, \cdots, r$ we have $v\left(y_{i}^{\prime} y_{1} / y_{i} y_{1}^{\prime}\right)=0$, so that by (1) and (4) we cannot have $v\left(y_{i}\right)=0$.

Example 10 becomes a little more transparent if we take $c_{1}=1$, write $y_{1}=x, y_{2}=x^{c_{2}}, \cdots, y_{r}=x^{c_{r}}$, and use Example 5 to alter the derivation of $K$ by multiplication by the factor $1 / x$. We then get the following result: If $1, c_{2}, \cdots, c_{r} \in C$ are linearly independent over $\boldsymbol{Q}$, we have a differential field $C\left(x, x^{c_{2}}, \cdots, x^{c_{r}}\right)$ with constants $C$ and derivation given by $\left(x^{c}\right)^{\prime}=c x^{c-1}$ for any $c \in \boldsymbol{Z}+\boldsymbol{Z} c_{2}+\cdots+\boldsymbol{Z} c_{r}$, and for any isomorphic embedding $\tau$ of $\boldsymbol{Z}+\boldsymbol{Z} c_{2}+\cdots+\boldsymbol{Z} \boldsymbol{c}_{r}$ into an ordered abelian group we have a differential valuation $v$ of $C\left(x, x^{c_{2}}\right.$, $\left.\cdots, x^{c_{r}}\right)$ such that $v(x)=\tau(1)$ and $v\left(x^{c_{i}}\right)=\tau\left(c_{i}\right)$ for $i=2, \cdots, r$; furthermore we obtain in this way all differential valuations $v$ of $C\left(x, x^{c_{2}}, \cdots, x^{c} r\right)$ such that $v(x) \neq 0$. As an example, take $C=C$ and choose $c \in C, c \notin \boldsymbol{Q}$. Then there are differential valuations of the differential field $C\left(x, x^{c}\right)$ for which $x$ has positive value and $x^{c}$ either positive or negative value, which can be infinitely small or infinitely large with respect to the value of $x$. It is hard to make analytic sense out of this if $c \in \boldsymbol{R}$, but if, for example, $c=i=\sqrt{-1}$, we get just such a differential valuation of $C\left(x, x^{i}\right)$ by setting $x=e^{-t-i t^{2}}, x^{i}=e^{t^{2-i t}}$ and letting $t \rightarrow+\infty$ through real values, as in Example 2.

TheOREM 3. Let $K$ be a field, $\mathscr{C}$ and $k$ subfields of $K$ that are linearly disjoint over $C=\mathscr{C} \cap k$ and such that $K=\mathscr{C}(k)$, let $k$ have the structure of a differential field with subfield of constants $C$, and let $v$ be a differential valuation of $k$. Then there is a unique derivation on $K$ that extends that on $k$ and is trivial on $\mathscr{C}$, and this derivation has $\mathscr{C}$ as its subfield of constants, and there is a unique valuation of $K$ that extends $v$ and is trivial on $\mathscr{C}$, and this is a differential valuation of $K$ with the same value group as $k$.

A derivation of $K=\mathscr{C}(k)$ that extends the given derivation of $k$ and is trivial on $\mathscr{C}$ is uniquely determined by the rules for differentiating rational functions, so there is at most one such derivation. To show the existence of such a derivation of $K$, choose a transcendence basis $\left\{c_{i}\right\}_{i \in I}$ of $\mathscr{C}$ over $C$ and note that the linear disjointness of $\mathscr{C}$ and $k$ over $C$ implies that $\left\{c_{i}\right\}_{i \in I}$ are algebraically independent over $k$, so that the given derivation can be extended to a derivation of $k\left[\left\{c_{i}\right\}_{i \in I}\right]$ that annuls each $c_{i}$, hence to a derivation of $k\left(\left\{c_{i}\right\}_{i \in I}\right)$, hence to a derivation of the algebraic extension $K$ of this latter field, and this last derivation will annul each element of $\mathscr{C}$, since $\mathscr{C}$ is algebraic over $C\left(\left\{c_{i}\right\}_{i \in I}\right)$. Thus we have the existence and 
uniqueness of our extended derivation on $K$. Now consider the differential valuation $v$ of $k$. Any nonzero element $x$ of $\mathscr{C}[k]$ can be written as $x=\sum_{i=1}^{n} \xi_{i} u_{i}$, for some $n$, with each $\xi_{i} \in \mathscr{C}^{*}$ and each $u_{i} \in k^{*}$. Suppose $v\left(u_{1}\right) \leqq v\left(u_{2}\right), \cdots, v\left(u_{n}\right)$, and let $a_{2}, \cdots, a_{n} \in C$ be such that $v\left(\left(u_{i} / u_{1}\right)-a_{i}\right)>0$ for $i=2, \cdots, n$. Then

$$
x=\left(\xi_{1}+a_{2} \xi_{2}+\cdots+a_{n} \xi_{n}\right) u_{1}+\sum_{i=2}^{n} \xi_{2}\left(u_{i}-a_{i} u_{1}\right),
$$

and $u_{i}-a_{i} u_{1} \in k$ with $v\left(u_{i}-a_{i} u_{1}\right)>v\left(u_{1}\right)$ for $i=2, \cdots, n$. Had $n$ been chosen minimal for our given $x$, we would have $\xi_{1}+a_{2} \xi_{2}+$ $\cdots+a_{n} \xi_{n} \neq 0$. Thus any nonzero $x \in \mathscr{C}[k]$ is of the form $x=\sum_{i=1}^{n} \xi_{i} u_{i}$, with each $\xi_{i} \in \mathscr{C}^{*}$, each $u_{i} \in k^{*}$, and with $v\left(u_{1}\right)<v\left(u_{2}\right)$, $\cdots, v\left(u_{n}\right)$. We claim that $v\left(u_{1}\right)$ depends only on $x$, and not on the choice of $n, \xi_{1}, \cdots, \xi_{n}, u_{1}, \cdots, u_{n}$. For otherwise we could obtain a relation $\sum_{i=1}^{n} \xi_{i} u_{i}=0$, with $n>0$, each $\xi_{i} \in \mathscr{C}^{*}$, each $u_{\imath} \in k^{*}$, and with $v\left(u_{1}\right)<v\left(u_{2}\right), \cdots, v\left(u_{n}\right)$, and we shall now show such a relation to be impossible. If $u_{2}, \cdots, u_{n}$ are linearly dependent over $C$ we can express one of these as a linear combination of the others with coefficients in $C$, and hence get a similar identity $\sum_{i=1}^{n} \xi_{i} u_{i}=0$, but with smaller $n$. We may therefore suppose $u_{2}, \cdots, u_{n}$ to be linearly independent over $C$. But $u_{1}, \cdots, u_{n}$ are linearly dependent over $\mathscr{C}$, hence by linear disjointness over $C$, so that $u_{1}$ is a linear combination of $u_{2}, \cdots, u_{n}$ with coefficients in $C$, contradicting $v\left(u_{1}\right)<v\left(u_{2}\right), \cdots$, $v\left(u_{n}\right)$. Thus $v\left(u_{1}\right)$ depends only on $x$. If $w$ is a valuation of $K$ that extends $v$ and is trivial on $\mathscr{C}$, then clearly $w(x)=v\left(u_{1}\right)$, so $w$ is uniquely determined on $\mathscr{C}[k]$, and hence on the quotient field of the latter ring, which is $K$. Conversely we may define $w$ on $\mathscr{C}[k]-\{0\}$ by $w(x)=v\left(u_{1}\right)$, and we get immediately that $w$ extends the map $v$, is zero on $\mathscr{C}^{*}$, and has the property that if $x_{1}, x_{2} \in \mathscr{C}[k]-\{0\}$, then $w\left(x_{1} x_{2}\right)=w\left(x_{1}\right)+w\left(x_{2}\right), w\left(x_{1}+x_{2}\right) \geqq \min \left\{w\left(x_{1}\right), w\left(x_{2}\right)\right\}$. Any element of $K^{*}$ can be written as $x / y$, with $x, y \in \mathscr{C}[k]-\{0\}$, and $w(x)-w(y)$ depends only on $x / y$, so that we may define $w(x / y)$ to be $w(x)-w(y)$, and thus we get our desired valuation on $K$. It remains only to show that the subfield of constants of $K$ is $\mathscr{C}$ and that $w$ is a differential valuation of $K$, and this is an immediate consequence of Theorem 2 applied to the present $K, k, \mathscr{C}$, and $C$, with $T=k^{*}$.

EXAMPLE 11. There is no differential valuation $v$ of the differential field of real-valued functions $\boldsymbol{R}(x, \cos x, \sin x)$ for which $v(x)<0$. For by Theorem 3 such a $v$ must extend to a differential valuation, also denoted $v$, of $\boldsymbol{C}(x, \cos x, \sin x)=\boldsymbol{C}\left(x, e^{i x}\right)$. Since $v\left(\left(e^{i x}\right)^{\prime} x / e^{i x} x^{\prime}\right)<0$, (4) implies that $v\left(e^{i x}\right) \neq 0$, so that the residue class field image of $\tan x$ is $\pm i$. 
THEOREM 4. Let $k$ be a differential field and let $v$ be a differential valuation of $k$ with value group $\Gamma$. Then there is a map i from $\Gamma^{*}=\Gamma-\{0\}$ into $\Gamma$ such that for all $a \in k^{*}$ with $v(a) \neq 0$ we have $\psi(v(a))=v\left(a^{\prime} / a\right)$, and $\psi$ has the following properties:

(a) If $\alpha \in \Gamma^{*}$ and $n \in Z, n \neq 0$, then $\psi(n \alpha)=\psi(\alpha)$.

(b) For any $\gamma \in \Gamma$, the set $\{\alpha \in \Gamma: \alpha=0$ or $\psi(\alpha) \geqq \gamma\}$ is a subgroup of $\Gamma$.

(c) For any $\alpha, \beta \in \Gamma^{*}, \psi(\beta)<\psi(\alpha)+|\alpha|$.

By Corollary 1 of Theorem 1 we have a well-defined map $\psi: \Gamma^{*} \rightarrow$ $\Gamma$ given by $\psi(v(a))=v\left(a^{\prime} / a\right)$ for any $a \in k^{*}$ such that $v(a) \neq 0$. If $n \in Z, n \neq 0$, then $\psi(n v(a))=\psi\left(v\left(a^{n}\right)\right)=v\left(\left(a^{n}\right)^{\prime} / a^{n}\right)=v\left(n a^{\prime} / a\right)=v\left(a^{\prime} / a\right)=$ $\psi(v(a))$, proving (a). Property (b) is verified by noting that if $a, b \in$ $k^{*}$ are such that $\psi(v(a)), \psi(v(b)) \geqq \gamma$, then $\psi(v(a)+v(b))=\psi(v(a b))=$ $v\left((a b)^{\prime} / a b\right)=v\left(\left(a^{\prime} / a\right)+\left(b^{\prime} / b\right)\right) \geqq \min \left\{v\left(a^{\prime} / a\right), v\left(b^{\prime} / b\right)\right\}=\min \{\psi(v(a))$, $\psi(v(b))\} \geqq \gamma$, provided all these symbols make sense, and also using the case $n=-1$ of (a) to get inverses. To prove (c), note first that we may take $\alpha>0$, since $\psi(\alpha)=\psi(-\alpha)$. Take $a, b \in k^{*}$ such that $v(a)=\alpha, v(b)=\beta$. Then $\psi(\alpha)+|\alpha|-\psi(\beta)=v\left(a^{\prime} / a\right)+v(a)-v\left(b^{\prime} / b\right)=$ $v\left(a^{\prime} b / b^{\prime}\right)>0$, by (1) of Theorem 1 .

It is convenient to recall here that for any abelian group $\Gamma$, the kernel of the homomorphism $\Gamma \rightarrow \Gamma \otimes_{Z} \boldsymbol{Q}$ which is given by $\gamma \mapsto \gamma \otimes 1$ is the torsion subgroup of $\Gamma$. Furthermore, the vector space over $\boldsymbol{Q}$ which is given by $\boldsymbol{Q} \Gamma=\Gamma \boldsymbol{\otimes}_{z} \boldsymbol{Q}$ is generated by the image of $\Gamma$ in $\boldsymbol{Q} \Gamma$. If $\Gamma$ is torsion free, we have an embedding of $\Gamma$ into the $\boldsymbol{Q}$ vector space $\boldsymbol{Q} \Gamma$. If $\Gamma$ is an ordered abelian group, then so is $\boldsymbol{Q} \Gamma$ in a natural way, and we have an order-preserving embedding of $\Gamma$ into $\boldsymbol{Q} \Gamma$.

In the most important applications of Theorem 4, the differential field $k$ will be differentially algebraic over its subfield of constants $C$ and finitely generated as a differential extension field of $C$, hence of finite transcendence degree over $C$. For any valuation $v$ of a field $k$ that that is trivial on the subfield $C$, the rational rank of $v$, i.e., $\operatorname{dim}_{Q} Q \Gamma$, where $\Gamma$ is the value group of $v$, is at most $\operatorname{deg} \operatorname{tr} k / C$ [4, p. 50]. It follows that in the case of Theorem 4 where $\operatorname{deg} \operatorname{tr} k / C$ is finite, the function $\psi$ is finitely valued, with $\#\left(\psi\left(\Gamma^{*}\right)\right) \leqq \operatorname{deg} \operatorname{tr} k / C$.

It will be shown in a later paper that if $\Gamma$ is any ordered abelian group and $\psi: \Gamma^{*} \rightarrow \Gamma$ a function satisfying (a), (b), (c), and if certain reasonable further conditions hold, such as $\psi$ being finitely valued, then $\Gamma$ and $\psi$ arise from a differential field with a differential valuation, as in Theorem 4.

ExAmPLE 12. Consider the Hardy field $k=\boldsymbol{R}\left(x, e^{x}, e^{x^{2}}, \log x\right)$ of real-valued functions as $x \rightarrow+\infty$, as in Example 1 . For any $N \in Z$, 
the limit as $x \rightarrow+\infty$ of each of the functions $\left(e^{x}\right)^{N} / e^{x^{2}}, x^{N} / e^{x}$, and $(\log x)^{N} / x$ is 0 . It follows that each nonzero element $f$ of $k$ can be written in one and only one way in the form

$$
f=\left(e^{-x^{2}}\right)^{n_{1}}\left(e^{-x}\right)^{n_{2}}(1 / x)^{n_{3}}(1 / \log x)^{n_{4}} f_{1},
$$

where $n_{1}, n_{2}, n_{3}, n_{4} \in \boldsymbol{Z}$ and $f_{1}$ has a finite nonzero limit as $x \rightarrow+\infty$. The map $f \mapsto\left(n_{1}, n_{2}, n_{3}, n_{4}\right)$ gives an isomorphism of the value group $\Gamma$ of $k$ with the group $\boldsymbol{Z}^{4}$, lexicographically ordered (i.e., $\left(n_{1}, n_{2}, n_{3}, n_{4}\right)>$ 0 if $n_{1}, n_{2}, n_{3}, n_{4}$ are not all zero and the first of these integers which is nonzero is positive). We compute immediately

$$
\psi\left(m_{1}, m_{2}, m_{3}, m_{4}\right)= \begin{cases}(0,0,-1,0) & \text { if } m_{1} \neq 0 \\ (0,0,0,0) & \text { if } m_{1}=0, \quad m_{2} \neq 0 \\ (0,0,1,0) & \text { if } m_{1}=m_{2}=0, m_{3} \neq 0 \\ (0,0,1,1) & \text { if } \quad m_{1}=m_{2}=m_{3}=0, \quad m_{4} \neq 0 .\end{cases}
$$

REMARK. As is illustrated by Example 12, in the case of Hardy fields, and also in the case of differential fields of germs of realvalued functions on deleted one-sided neighborhoods of a point of $\boldsymbol{R}$, we have the important principle

(*) Let $\alpha>\beta$ be positive elements of $\Gamma$. Then $\psi(\alpha) \leqq \psi(\beta)$. Indeed if $\alpha>n \beta$ for all positive integers $n$ then $\psi(\alpha)<\psi(\beta)$, and in the contrary case $\psi(\alpha)=\psi(\beta)$.

To prove this, suppose to fix ideas that we are working with a Hardy field $k$ of real-valued functions near $+\infty$. Choose $f, g \in k$ such that $\alpha=v(f), \beta=v(g)$. Then $f(x), g(x)$, and $f(x) / g(x)$ all have limit 0 as $x \rightarrow+\infty$. Assume, as we may, that $f(x), g(x)>0$. Then for large $x$ we have $f(x)<g(x)$, so $\log f(x)<\log g(x)$, or, since we are dealing with the logarithms of small positive numbers, $|\log f(x)|>|\log g(x)|$. Adjoining the logarithms of positive functions does not spoil a Hardy field (cf. [1, p. 113]), so we may assume that $\log f, \log g \in k$. Since $|\log f(x)|>|\log g(x)|$ for large $x$, we have $v(\log f) \leqq v(\log g)$, which implies $v\left((\log f)^{\prime}\right) \leqq v\left((\log g)^{\prime}\right)$. Therefore $v\left(f^{\prime} / f\right) \leqq v\left(g^{\prime} / g\right)$, or $\psi(\alpha) \leqq \psi(\beta)$. This proves the first statement. To prove the second statement, choose $f, g$ as above and apply the given argument to $f$ and $g^{n}$. We get $|\log f(x)|>|n \log g(x)|$, or $|\log f(x) / \log g(x)|>n$ for large $x$. Since this is true for all $n \in Z$, we have $v(\log f / \log g)<0$, so $v(\log f)<v(\log g)$, so $v\left(f^{\prime} / f\right)<v\left(g^{\prime} / g\right)$, or $\psi(\alpha)<\psi(\beta)$. This proves the second statement. Finally, if there is a positive integer $n$ such that $\alpha<n \beta$, then $\psi(\alpha) \leqq \psi(\beta)=\psi(n \beta) \leqq$ $\psi(\alpha)$, so $\psi(\alpha)=\psi(\beta)$. 
EXAMPLE 13. The statement $\left(^{*}\right)$ of the above Remark is not valid in general, not even in the case of differential valuations associated with differential fields of meromorphic functions which approach limits along a curve in $C$, as in Example 2. For example, consider the differential field of meromorphic functions of the complex variable $z$ given by $k=C\left(z, e^{z}, e^{z^{2}}\right)$, whose derivation is the usual differentiation with respect to $z$. If we set $z(t)=t^{2}+i\left(t^{2}-1 / t\right)$ and let $t \rightarrow+\infty$ through real values, we get

$$
\lim _{t \rightarrow+\infty} \frac{z(t)}{t^{2}}=1+i,\left|e^{z(t)}\right|=e^{t^{2}}, \lim _{t \rightarrow+\infty} \frac{\left|e^{(z(t))^{2}}\right|}{e^{2 t}}=1 .
$$

For any $N \in \boldsymbol{Z}$, both $\left(e^{z^{2}}\right)^{N} / e^{z}$ and $z^{N} / e^{z^{2}}$ approach 0 as $z \rightarrow \infty$ along the curve $z=z(t), t \rightarrow+\infty$. It follows that any nonzero element $f \in k$ can be written in one and only one way in the form

$$
f=\left(e^{-z}\right)^{n_{1}}\left(e^{-z^{2}}\right)^{n_{2}}(1 / z)^{n_{3}} f_{1},
$$

where $n_{1}, n_{2}, n_{3} \in Z$ and $f_{1}$ has a nonzero limit in $C$ as $z \rightarrow \infty$ along the curve $z=z(t), t \rightarrow+\infty$. Thus we have a valuation of $k$, and the map $f \mapsto\left(n_{1}, n_{2}, n_{3}\right)$ is an isomorphism of the value group $\Gamma$ with the lexicographically ordered group $\boldsymbol{Z}^{3}$. We see by the method of Example 2 or by Theorem 2 that our valuation is a differential valuation. We compute $\psi(1,0,0)=v\left(\left(e^{-z}\right)^{\prime} / e^{-z}\right)=0, \psi(0,1,0)=$ $v\left(\left(e^{-z^{2}}\right)^{\prime} / e^{-z^{2}}\right)=(0,0,-1), \psi(0,0,1)=v\left((1 / z)^{\prime} /(1 / z)\right)=(0,0,1)$. We get a contradiction to $(*)$ by taking $\alpha=(1,0,0), \beta=(0,1,0)$.

The following theorem will turn out to be of fundamental importance.

THeOREM 5. Let $\Gamma$ be an ordered abelian group, $\Gamma^{*}=\Gamma-\{0\}$, and ' : $\Gamma^{*} \rightarrow \Gamma$ a map such that

(a) if $\alpha \in \Gamma^{*}$ and $n \in Z, n \neq 0$, then $\psi(n \alpha)=\psi(\alpha)$

(b) for any $\gamma \in \Gamma$, the set $\{\alpha \in \Gamma: \alpha=0$ or $\psi(\alpha) \geqq \gamma\}$ is a subgroup of $\Gamma$

(c) for any $\alpha, \beta \in \Gamma^{*}, \psi(\beta)<\psi(\alpha)+|\alpha|$. Then for any $\alpha, \beta \in \Gamma^{*}$ and any positive integer $n$,

$$
n(\psi(\beta)-\psi(\alpha))<|\alpha|
$$

We must show that for any specific $\alpha_{0}, \beta_{0} \in \Gamma^{*}$ and integer $n_{0}>0$ we have $n_{0}\left(\psi\left(\beta_{0}\right)-\psi\left(\alpha_{0}\right)\right)<\left|\alpha_{0}\right|$. It suffices to consider only the case $\psi\left(\beta_{0}\right)>\psi\left(\alpha_{0}\right)$. Replacing $\psi$, if necessary, by the map $\psi_{1}: \Gamma^{*} \rightarrow \Gamma$ given by $\psi_{1}(\gamma)=\psi(\gamma)-\psi\left(\alpha_{0}\right)$, which also satisfies conditions (a), (b), (c), we are reduced to consideration of the case $\psi\left(\alpha_{0}\right)=0, \psi\left(\beta_{0}\right)>0$. Now consider the function $\psi_{2}: \Gamma^{*} \rightarrow \Gamma$ defined by $\psi_{2}(\gamma)=0$ if $\psi(\gamma) \leqq$ $0, \psi_{2}(\gamma)=\psi\left(\beta_{0}\right)$ if $\psi(\gamma)>0$. We verify immediately that $\psi_{2}$ also 
satisfies conditions (a), (b), (c), and that $\psi_{2}$ and $\psi$ agree on $\alpha_{0}$ and $\beta_{0}$. Thus we are reduced to considering the special case of the theorem in which there is a positive $\delta \in \Gamma$ such that $\psi\left(\Gamma^{*}\right)=\{0, \delta\}$, and here we have to prove that if $\alpha \in \Gamma^{*}$ and $\psi(\alpha)=0$, then $n \delta<|\alpha|$ for any positive integer $n$. Now since $\delta<\psi(\delta)+|\delta|$, we cannot have $\psi(\delta)=$ 0 . Hence $\psi(\delta)=\delta$. To prove for some fixed $\alpha \in \Gamma^{*}$ such that $\psi(\alpha)=$ 0 that $n \delta<|\alpha|$ for any positive integer $n$, it suffices to restrict our attention to the subgroup of $\Gamma$ given by $\boldsymbol{Z} \alpha+\boldsymbol{Z} \delta$. Noting that $\psi\left((\boldsymbol{Z} \alpha+\boldsymbol{Z} \delta)^{*}\right)=\{0, \delta\}$, we are reduced to the special case where $\Gamma=\boldsymbol{Z} \alpha+\boldsymbol{Z} \delta$. Since $\psi$ takes on two values on $\Gamma^{*}, \Gamma$ cannot be cyclic, by condition (a). Hence $\Gamma$ is a free abelian group on two generators. The function $\psi$ takes on the value $\delta$ on $(\boldsymbol{Z} \delta)^{*}$, and the value 0 elsewhere on $\Gamma^{*}$. If the ordering of $\Gamma$ is archimedian, $\Gamma$ is orderisomorphic to a subgroup of $\boldsymbol{R}$, hence has arbitrarily small positive elements. Thus if $\Gamma$ is archimedian there exists $\beta \in \Gamma$ such that $0<\beta<\delta$, and we get $\beta \notin \boldsymbol{Z} \delta$, so that $\psi(\beta)=0$, and hence we have a contradiction in the statement $\delta=\psi(\delta)<\psi(\beta)+|\beta|$. Thus the ordering of $\Gamma$ is not archimedian. Therefore $\Gamma$ contains a proper convex (or "isolated") subgroup, necessarily of rank 1, therefore cyclic with some generator $\varepsilon>0$, and $\varepsilon$ is the smallest positive element of $\Gamma$. Now $\delta<\psi(\varepsilon)+\varepsilon$ so $0 \leqq \delta-\psi(\varepsilon)<\varepsilon$, and therefore $\delta=\psi(\varepsilon)$. Therefore $\psi$ equals $\delta$ on $(\boldsymbol{Z} \varepsilon)^{*}$ and equals 0 elsewhere on $\Gamma^{*}$, so that $\delta \in Z \varepsilon$. Since the image of $|\alpha|$ in the ordered abelian group $\Gamma / Z \varepsilon$ (which is isomorphic to $Z$ ) is positive, we have $|\alpha|>n \varepsilon$ for all $n \in Z$, and this completes the proof.

Lemma. Let $k$ be a differential field, $v$ a differential valuation of $k, p$ a prime number and let $u \in k^{*}$ be such that $v(u) \notin p v\left(k^{*}\right)$. Then there is a unique extension (to within order-isomorphism) of the valuation $v$ to $k\left(u^{1 / p}\right)$ and this extended valuation is a differential valuation of $k\left(u^{1 / p}\right)$ which has the same constants as $k$.

Since $v(u) \notin p v\left(k^{*}\right)$, we have $u^{1 / p} \notin k$, so that $\left[k\left(u^{1 / p}\right): k\right]=p$ and $1, u, \cdots, u^{(p-1) / p}$ are a $k$-basis for $k\left(u^{1 / p}\right)$. If $w$ is any extension to $k\left(u^{1 / p}\right)$ of the valuation $v$ of $k$ and $a_{0}, a_{1}, \cdots, a_{p-1} \in k$, then $w\left(a_{i} u^{i / p}\right) \neq$ $w\left(a_{j} u^{j / p}\right)$ if $a_{i}, a_{j} \neq 0$ and $i \not \equiv j(\bmod p)$. Thus

$$
w\left(\sum_{i=0}^{p-1} a_{i} u^{i / p}\right)=\min _{i=0, \cdots, p-1} w\left(a_{i} u^{i / p}\right)=(1 / p) \min _{i=0, \cdots, p-1} v\left(a_{i}^{p} u^{i}\right) .
$$

Therefore $w$ is completely determined by $v$, so that we can simplify our notation by writing $w=v$. We now try to apply Theorem 2 to the case of $K=k\left(u^{1 / p}\right)$, with the present $k$ and $v$, with $\mathscr{C}=C=$ constants of $k$, and with $T=\bigcup_{i=0, \cdots, p-1} k^{*} u^{i / p}$. That $k^{*} \subset T$ and $K=$ 
$\mathscr{C}(T)$ are clear. If $a \in k^{*}, i=1, \cdots, p-1$, and $a u^{i / p}$ is a constant, then so is $a^{p} u^{i} \in k$, so that $a^{p} u^{i} \in C$, which contradicts $v(u) \notin p v\left(k^{*}\right)$. Thus any constant in $T$ is in $k$, hence in $C$. The condition $(a \in T$, $v(a)=0) \Longrightarrow\left(a \in k^{*}\right)$ is trivial. If $a \in T$ then $a^{p} \in k^{*}$ and $\left(a^{p}\right)^{\prime} / a^{p}=$ $p a^{\prime} / a$, so that $v\left(a^{\prime} / a\right)=v\left(\left(a^{p}\right)^{\prime} / a^{p}\right)$. Hence if $a, b \in T$ and $v(a), v(b)>0$ we have $v\left(a^{\prime} b / b^{\prime}\right)=v\left(a^{\prime} / a\right)-v\left(b^{\prime} / b\right)+v(a)=v\left(\left(a^{p}\right)^{\prime} / a^{p}\right)-v\left(\left(b^{p}\right)^{\prime} / b^{p}\right)+$ $v\left(a^{p}\right) / p$. But this last expression is positive, by Theorem 5, since $a^{p}, b^{p} \in k$ and $v$ is a differential valuation of $k$. Thus Theorem 2 is applicable, and this completes the proof.

THEOREM 6. Let $k$ be a differential field, $v$ a differential valuation of $k$. Then any extension of $v$ to a valuation of an algebraic extension field of $k$ is a differential valuation of the extension field.

It suffices to consider the case where the extension field is the algebraic closure $\bar{k}$ of $k$. Before proceeding with the proof, two remarks are appropriate. First, recall the elementary fact that the derivation on $k$ extends in a unique way to a derivation on $\bar{k}$. Thus the extended derivation on $\bar{k}$ commutes with each $k$-automorphism of $\bar{k}$. Second, recall that the valuation $v$ of $k$ can be extended to a valuation of $\bar{k}$, and that (except for an order-isomorphism of the value groups) any two extensions of $v$ to $\bar{k}$ differ by a $k$-automorphism of $\bar{k}$ (cf. [4, p. 28]). In what follows we denote by the same symbol $v$ a fixed extension to $\bar{k}$ of the given valuation of $k$, and we go about proving that $v$ is a differential valuation of $\bar{k}$. The proof uses a number of standard ideas, appropriately modified, that may be found in [3, Chap. 2]. We first note that each subfield of $\bar{k}$ that contains $k$ is itself a differential field and we partially order by inclusion the set of those intermediate fields on which the restriction of $v$ is a differential valuation. By Zorn's lemma, there is a maximal such intermediate field. Replacing $k$ by this maximal intermediate field if necessary, we are reduced to the case where the restriction of $v$ to $k$ is a differential valuation, but the restriction of $v$ to any subfield of $\bar{k}$ that properly contains $k$ is not, and we have to show that in this case $\bar{k}=k$. Let $C, \mathscr{C}$ be respectively the subfields of constants of $k$ and of $\bar{k}$. Then $\mathscr{C}$ is the algebraic closure of $C$ in $\bar{k}$, hence is itself algebraically closed, and since $v$ is trivial on $C$ it is also trivial on $\mathscr{C}$. Theorem 3 implies that the restriction of $v$ to $\mathscr{C}(k)$ is a differential valuation. Thus $\mathscr{C}(k)=k$, and hence $\mathscr{C}=C$. In particular, $C$ is algebraically closed. When we extend a valuation of a field to an algebraic extension field, the residue class field of the extension field is algebraic over the residue class field of the original field. Thus the residue class field of $\bar{k}$ is algebraic over 
that of $k$, which is $C$; thus $\bar{k}$ and $k$ have the same residue class field $C$. When we extend a valuation of a field to a valuation of an algebraic extension field, the value group of the extension field contains as a subgroup the value group of the small field, with the quotient group a torsion group. Let $\Gamma$ be the value group of $k$. The lemma tells us that for any prime number $p, \Gamma=p \Gamma$. Thus $\Gamma$ is a divisible group, that is $\Gamma=\boldsymbol{Q} \Gamma$, so that $\Gamma$ is also the value group of $\bar{k}$. Thus $\bar{k}$ and $k$ have the same residue class field $C$ and the same value group $\Gamma$. Supposing that $k \neq \bar{k}$, we fix a field $K$ such that $k \subset K \subset \bar{k}$ and $n=[K: k]>1$ is minimal. Next fix some $\alpha \in K, \alpha \notin k$. Then $K=k(\alpha)$. We define a subset $S$ of $\Gamma$ by

$$
S=\{\gamma \in \Gamma: \text { for some } a \in k, v(\alpha-a)=\gamma\} .
$$

Fix a function $\gamma \mapsto a_{\gamma}$ from $S$ into $k$ such that $v\left(\alpha-a_{\gamma}\right)=\gamma$ for each $\gamma \in S$. The set $S$ is nonempty. If $\gamma \in S$, we can find $b \in k$ such that $v(b)=\gamma$, so that $v\left(\left(\alpha-a_{r}\right) / b\right)=0$, and then we can find $c \in C$ having the same residue class field image as $\left(\alpha-a_{r}\right) / b$. Then $v\left(\left(\alpha-a_{r}\right) / b-c\right)>0$. Thus $a_{r}+b c \in k$ and $v\left(\alpha-\left(a_{r}+b c\right)\right)>\gamma$. Thus $S$ does not have a greatest element. Now for any $\xi \in \bar{k}$, consider $v\left(a_{\gamma}-\xi\right)$ as $\gamma$ ranges over $S$. This quantity is defined except when $a_{r}=\xi$, which can happen for at most one $\gamma \in S$, and then only if $\xi \in k$. We have $v\left(a_{\gamma}-\xi\right)=v\left(\left(a_{\gamma}-\alpha\right)+(\alpha-\xi)\right)$. It may be that for some $\sigma \in S$ we have $v(\alpha-\xi) \leqq \sigma$, and then for $\gamma \in S, \gamma>\sigma$ we have $v\left(a_{\gamma}-\xi\right)=v\left(\left(a_{\gamma}-\alpha\right)+(\alpha-\xi)\right)=v(\alpha-\xi)$. In this case $v\left(a_{\gamma}-\xi\right)$ takes on the fixed value $v(\alpha-\xi)$ for all sufficiently large $\gamma \in S$ (this happens, for example, if $\xi \in k)$. On the other hand, we may have $v(\alpha-\xi)$ greater than each element of $S$. In this case, for each $\gamma \in S$ we have $v\left(a_{\gamma}-\xi\right)=v\left(\left(a_{\gamma}-\alpha\right)+(\alpha-\xi)\right)=v\left(a_{\gamma}-\alpha\right)=\gamma$, so $v\left(a_{\gamma}-\xi\right)$ increases with $\gamma$ for $\gamma \in S$ (this happens, for example, if $\xi=\alpha$ ). Now let $x$ be an indeterminate over $k$ and let $f(x) \in k[x]$ be a nonzero polynomial. Writing $f(x)$ as the product of an element of $k$ and several monic linear factors $x-\xi$, with $\xi \in \bar{k}$, we see that either $v\left(f\left(a_{r}\right)\right)=v(f(a))$ if $\gamma \in S$ is sufficiently large, or $v\left(f\left(a_{r}\right)\right)$ is a strictly increasing function of $\gamma$ if $\gamma \in S$ is sufficiently large. For example, if $f(x) \in k[x]$ is linear, we have $v\left(f\left(a_{\gamma}\right)\right)=v(f(\alpha))$ for all sufficiently large $\gamma \in S$, while if $\phi(x)$ is the minimal polynomial of $\alpha$ over $k$, we have $v\left(\phi\left(a_{r}\right)\right)$ increasing for all sufficiently large $\gamma \in S$. By the minimality property of $n=[k(\alpha): k]=\operatorname{deg} \phi$, any nonzero $f(x) \in k[x]$ of degree less than $n$ factors into linear factors in $k[x]$, so that for such an $f(x)$ we have $v\left(f\left(a_{r}\right)\right)=v(f(\alpha))$ for all sufficiently large $\gamma \in S$. Write $\phi(x)=\sum_{i=0}^{n} a_{i} x^{i}$, with each $a_{i} \in k$, and set $\phi_{0}(x)=\sum_{i=0}^{n} a_{i}^{\prime} x^{i}, \phi^{\prime}(x)=$ $\sum_{i=0}^{n} i a_{i} x^{i-1}$, both nonzero elements of $k[x]$ of degree less than $n$. Then $0=(\phi(\alpha))^{\prime}=\phi_{0}(\alpha)+\alpha^{\prime} \phi^{\prime}(\alpha)$, so that $\alpha^{\prime}=-\phi_{0}(\alpha) / \phi^{\prime}(\alpha)$. Hence 
$v\left(\alpha^{\prime}\right)=v\left(\phi_{0}(\alpha)\right)-v\left(\phi^{\prime}(\alpha)\right)=v\left(\phi_{0}\left(a_{r}\right)\right)-v\left(\phi^{\prime}\left(a_{r}\right)\right)=v\left(\phi_{0}\left(a_{r}\right) / \phi^{\prime}\left(a_{r}\right)\right)$ if $\gamma \in S$ is sufficiently large. We also have, for each $\gamma \in S$,

$$
\left(\phi\left(a_{\gamma}\right)\right)^{\prime}=\phi_{0}\left(a_{\gamma}\right)+a_{,}^{\prime} \phi^{\prime}\left(a_{\gamma}\right) \text {. }
$$

For $\gamma \in S$ sufficiently large, we have $v\left(\phi_{0}\left(a_{r}\right)\right)=v\left(\phi_{0}(\alpha)\right)$ and $v\left(\phi^{\prime}\left(a_{r}\right)\right)=$ $v\left(\phi^{\prime}(\alpha)\right)$. We also know that $v\left(a_{\gamma}\right)=v(\alpha)$ for sufficiently large $\gamma \in S$, so that if $v(\alpha) \neq 0$ then $v\left(a_{\gamma}^{\prime}\right)$ is constant for sufficiently large $\gamma \in S$. But $v\left(\left(\phi\left(a_{r}\right)\right)^{\prime}\right)$ is an increasing function of $\gamma$ for sufficiently large $\gamma \in S$, since $v\left(\phi\left(a_{\gamma}\right)\right)$ is an increasing function of $\gamma$ and $v$ is a differential valuation on $k$. If $v(\alpha) \neq 0$ this can happen only if for sufficiently large $\gamma \in S$ we have $v\left(\phi_{0}\left(a_{r}\right)\right)=v\left(a_{r}^{\prime} \phi^{\prime}\left(a_{r}\right)\right)$, in which case $v\left(a_{r}^{\prime}\right)=v\left(\phi_{0}\left(a_{r}\right) / \phi^{\prime}\left(a_{r}\right)\right)=v\left(\alpha^{\prime}\right)$. Thus for any $\alpha \in K$ such that $\alpha \notin k$ and $v(\alpha) \neq 0$, we can find an element $a \in k$ such that $v(\alpha)=v(a)$ and $v\left(\alpha^{\prime}\right)=v\left(a^{\prime}\right)$. This last statement is also true, trivially, if $\alpha \in k$, $v(\alpha) \neq 0$. Since $v$ is a differential valuation on $k$, property (5) of Corollary 1 to Theorem 1 holds for elements of $k$, hence, by what we have just shown, for elements of $K$. The converse part of the same Corollary 1 now implies that $v$ is a differential valuation on $K$. This contradicts the maximality assumption we have made on $k$, showing that $k=\bar{k}$ and completing the proof.

It will be shown in another paper that any differential valuation of a differential field $k$ can be extended to a differential valuation of any liouvillian extension field of $k$.

\section{REFERENCES}

1. N. Bourbaki, Fonctions d'une Variable Réele, Chap. V (Étude Locale des Fonctions), 2nd ed., Hermann, Paris, 1961.

2. E. Kolchin, Rational approximation to solutions of algebraic differential equations, Proc. Amer. Math. Soc., 10 (1959), 238-244.

3. O. Schilling, The Theory of Valuations, Math. Surveys IV, Amer. Math. Soc., New York, 1950.

4. O. Zariski and P. Samuel, Commutative Algebra, vol. II, Van Nostrand, New York, 1960.

Received September, 21, 1978. Research supported by National Science Foundation grant number MCS77-03719 A02.

UNIVERSITY OF CALIFORNIA

BERKELEY, CA 94720 



\section{PACIFIC JOURNAL OF MATHEMATICS}

\section{EDITORS}

DONALD BABBITT (Managing Editor)

University of Galifornia

Los Angeles, California 90024

HUGo RossI

University of Utah

Salt Lake City, UT 84112

C. C. MOORE AND ANDREW OGG

University of California

Berkeley, CA 94720
J. DUGUNDJI

Department of Mathematics University of Southern California Los Angeles, California 90007

R. FINN AND J. MILGRAM Stanford University Stanford, California 94305

\section{ASSOCIATE EDITORS}

E. F. BECKENBACH

B. H. NEUMANN

F. WOLF

K. YosHIDA

\section{SUPPORTING INSTITUTIONS}

UNIVERSITY OF BRITISH COLUMBIA CALIFORNIA INSTITUTE OF TECHNOLOGY UNIVERSITY OF CALIFORNIA MONTANA STATE UNIVERSITY UNIVERSITY OF NEVADA, RENO NEW MEXICO STATE UNIVERSITY OREGON STATE UNIVERSITY UNIVERSITY OF OREGON
UNIVERSITY OF SOUTHERN CALIFONIA STANFORD UNIVERSITY UNIVERSITY OF HAWAII UNIVERSITY OF TOKYO UNIVERSITY OF UTAH WASHINGTON STATE UNIVERSITY UNIVERSITY OF WASHINGTON 


\section{Pacific Journal of Mathematics}

\section{Vol. 86, No. $1 \quad$ November, 1980}

Gert Einar Torsten Almkvist, Invariants, mostly old ones .............. 1

Hyman Bass, Groups of integral representation type ................ 15

A. Białynicki-Birula, On action of SL(2) on complete algebraic

varieties........................................

Frederick Paul Greenleaf and Martin Allen Moskowitz, Groups of

automorphisms of Lie groups: density properties, bounded orbits, and

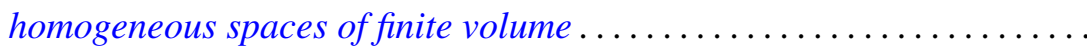

Raymond Taylor Hoobler, A cohomological interpretation of Brauer groups

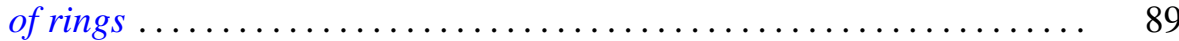

Irving Kaplansky, Superalgebras ........................ 93

Jerrold Lewis Kleinstein and Alex I. Rosenberg, Succinct and

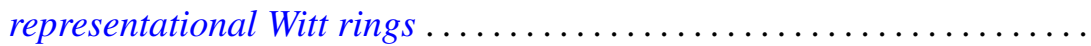

E. R. Kolchin, On universal extensions of differential fields ............ 139

Andy R. Magid, Analytic subgroups of affine algebraic groups. II ....... 145

Calvin Cooper Moore, The Mautner phenomenon for general unitary

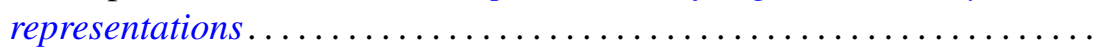

George Daniel Mostow, On a remarkable class of polyhedra in complex hyperbolic space ................................ 171

Brian Lee Peterson, Extensions of pro-affine algebraic groups. II . . . . . . 277

John Henry Reinoehl, Lie algebras and affine algebraic groups......... 287

Maxwell Alexander Rosenlicht, Differential valuations .

John Brendan Sullivan, The second Lie algebra cohomology group and Weyl modules..................................... 321

Moss Eisenberg Sweedler, Right derivations and right differential

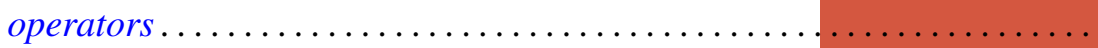

Bostwick Frampton Wyman, Time varying linear discrete-time systems. II. Duality.................................. 